(polyvinyl chloride) plastic products, including some medical devices such as IVs, blood bags, and the tubing used for dialysis, respiratory therapy, heart-bypass machines, and nasogastric feedings.

Although the current risks associated with DEHP are based on animal studies and apparent adverse developmental effects on the testicles or the production of normal sperm in young animals, the FDA cautions that very young male infants who are critically ill and have prolonged exposure to multiple devices containing DEHP may be at risk also.

Additional information on the human health risks of exposure to medical devices containing DEHP and on the FDA's efforts to assess and communicate these risks to healthcare providers adequately is available on the Internet at http://www.fda.gov/cdrh/ocd/dehp.html.

\section{MRSA in Rural US}

Until recently, methicillin-resistant Staphylococcus aureus (MRSA) infections were acquired primarily in nosocomial settings. In 1999, four deaths among children in Minnesota and North Dakota, one of which occurred in an American Indian, were attributed to community-acquired MRSA infection. These findings suggested that MRSA was being acquired outside nosocomial settings. Groom and colleagues conducted a study to document the occurrence of community-acquired MRSA infections and to evaluate risk factors for community-acquired MRSA infection compared with methicillin-susceptible $S$ aureus (MSSA) infection.

This retrospective cohort study, using medical record review, was conducted in an Indian Health Service facility in a rural Midwestern American Indian community and included patients whose medical records indicated laboratory-confirmed $S$ aureus infection diagnosed during 1997. The primary outcome measure was the proportion of MRSA infections classified as community-acquired, based on standardized criteria; risk factors for communityacquired MRSA infection, compared with those for community-acquired MSSA infection; and relatedness of MRSA strains, determined by pulsed-field gel electrophoresis (PFGE). The results indicated that, of $112 S$ aureus isolates, $62(55 \%)$ were MRSA and 50 (45\%) were MSSA. Forty-six (74\%) of the 62 MRSA infections were classified as community-acquired. Risk factors for community-acquired MRSA infections were not significantly different from those for community-acquired MSSA. PFGE subtyping indicated that $34(89 \%)$ of 38 community-acquired MRSA isolates were clonally related and distinct from nosocomial MRSA isolates found in the region.

The authors concluded that community-acquired MRSA may have replaced community-acquired MSSA as the dominant strain in this community. Antimicrobial-susceptibility patterns and PFGE subtyping support the finding that MRSA is circulating beyond nosocomial settings in this, and possibly in other, rural US communities. Based on these findings, the authors recommended that healthcare practitioners in rural communities in the Midwest consider the possibility of
MRSA infection among young, healthy patients without a history of nosocomial exposure. Obtaining cultures of suspected $S$ aureus infections and conducting antibiotic-susceptibility testing, particularly in communities with known high rates of MRSA infection, are important measures to ensure that appropriate antibiotic therapy is provided.

FROM: Groom AV, Wolsey DH, Naimi TS, Smith K, Johnson S, Boxrud D, et al. Community-acquired methicillin-resistant Staphylococcus aureus in a rural American Indian community. JAMA 2001;286:1201-1205.

\section{FDA Approves First Nucleic Acid Test for $\mathrm{HIV}$ and $\mathrm{HCV}$}

The FDA licensed the first nucleic acid test (NAT) systems intended for screening of plasma donors. These test systems are expected to ensure further the safety of plasmaderived products by permitting earlier detection of human immunodeficiency virus (HIV) and hepatitis C virus (HCV) infections in donors. The newly approved test systems were developed by National Genetics Institute, Los Angeles, for screening plasma used in manufacturing of products such as clotting factors and immune globulins. Alpha Therapeutic Corporation, Los Angeles, also was approved to use new testing systems at its plasma collection facilities.

NAT is a recently developed technology that allows detection of very small amounts of genetic material (DNA or RNA) by a process of massive copying (amplification) of a gene fragment. The approved test systems permit highly sensitive detection of RNA from HIV, type 1 (the HIV variant that is responsible for the vast majority of acquired immunodeficiency virus (AIDS) cases in the United States), and HCV in test pools of 512 plasma samples obtained from multiple donors. The use of pooled plasma samples for testing makes use of the NAT system cost-effective. However, if a test pool is positive for either virus, the individual donation that is suspected of containing a virus can be identified and would not be used for further manufacturing, and the donor can be deferred and notified.

Although effective procedures for virus inactivation are required in the manufacturing of all US-licensed plasma derivatives, removal of potentially infectious donations through donor screening adds a safeguard by limiting the amount of virus contamination that the manufacturing process must clear. Currently, donors of blood and plasma are tested for antibodies to HCV, antibodies to HIV, and HIV-1 antigens, which are the virus's own proteins. However, there is still a "window period" during which a donor can be infected but have negative screening tests. With the use of NAT for HCV, the window period for detection of $\mathrm{HCV}$ is reduced by 57 days (from an average of 82 days to 25 days). For HIV-1, the average window period with antibody tests is 22 days. Antigen testing cuts the window period to approximately 16 days, and NAT further reduces this period to 12 days.

FROM: FDA Press Release. FDA approves first nucleic acid test (NAT) systems to screen plasma for human immunodeficiency virus (HIV) and hepatitis $\mathrm{C}$ virus 
(HCV). September 21, 2001. http://www.fda.gov/bbs/ topics/ANSWERS/2001/ANS01103.html.

\section{Reuse of Single-Use Devices: FDA Delays Enforcement}

Hospitals that reprocess single-use devices (SUDs) have been given an extension on the date when the FDA will begin enforcing its new reprocessing requirements covering medical-device reporting, tracking, corrections and removals, quality system, and labeling. The extended deadline is August 14, 2002.

Hospitals still are required to register with the FDA and to list the SUDs they plan to reprocess. The FDA shortly will begin inspecting hospitals to assess their compliance with the requirements, but it intends to use those inspections as an opportunity to educate hospitals in the new requirements rather than to enforce actions. The education policy will be in effect until August 14, 2002 , "provided that the hospitals are taking steps to correct the violations noted in the inspection and that the violations do not pose a serious public health threat."

FROM: AHA news online; October 11, 2001. http:// www.AHA.org.

FDA, Center for Devices and Radiological Health. Changes in enforcement of FDA's requirements on reprocessing of single-use devices. http://www.fda.gov/cdrh/ reuse/reuse-letter-092501.html.

\section{Reuse of Single-Use Devices Not Widespread}

Two recent surveys of hospitals found that reuse of single-use devices is not widespread and that the majority of hospitals use third-party reprocessors. Nearly one half $(47.8 \%)$ of all respondents to an ECRI e-mail survey on single-use device (SUD) reuse reported that they will not allow any reuse in light of last year's FDA regulations. Most respondents did not change their decisions on reuse as the August 14, 2001, enforcement deadline approached; those who did change generally were less willing to reuse devices than they had been previously. Most facilities (86.4\%) that plan to continue reuse will do so exclusively through third-party reprocessors.

In a recent study completed by JCAHO for the FDA, hospitals reported reusing medical devices intended for single use at a relatively low rate. Of the 800 hospitals polled, $11 \%$ reuse SUDs and only $2 \%$ reuse class III devices posing the highest risk to patient safety. The most commonly used class III devices are electrodes used during angioplasty for coronary artery disease. Other reused class III devices include endotracheal tubes and angioplasty catheters. The report shows that $80 \%$ of hospitals reusing SUDs rely on commercial vendors registered and listed with the FDA to reprocess these devices. The study was conducted between September 2000 and March 2001 at hospitals of all sizes.
FROM: JCAHOnline. August 2001. http://www. jcaho.org/tip/jonline0801.html.

\section{Infection Rates in Low-Birth-Weight Neonates}

Zafar and coinvestigators from St Louis conducted a study to determine factors associated with an increasing rate of nosocomial infections (NIs) in infants with very low birth weights. They performed a retrospective review of clinical and NI databases for all infants with birth weights of $\leqslant 1,500 \mathrm{~g}$ admitted to an academic neonatal intensive care unit between January 1, 1991, and December 31, 1997 $(n=1,184)$. Two study periods were compared: 1991 to 1995 and 1996 to 1997. Among the 1,085 infants who survived beyond 48 hours, the proportion who developed NIs increased from $22 \%$ to $31 \%(P=.001)$, and the infection rate increased from 0.5 to 0.8 per 100 patient-days $(P<.001)$ during the period from 1996 to 1997. During that same period, the median duration of indwelling vascular access increased from 10 to 16 days $(P<.001)$, and the median duration of mechanical ventilation increased from 7 to 12 days $(P<.001)$. Although the device-specific rate of bloodstream or respiratory infections did not change, the increase in infections was directly attributable to the increasing proportion of infants who required these devices. In both study periods, the peak incidence of initial infection occurred between 10 and 20 days of age. For the entire sample, proportional hazard models identified birth weight, duration of vascular access, and postnatal corticosteroid exposure as significant contributors to the risk of infection.

The authors concluded that the increasing number of technology-dependent infants was the primary determinant in the increase of NIs. Because these infections occur in a small proportion of infants, understanding the host factors that contribute to this vulnerability is necessary to control NIs in neonatal intensive care units.

FROM: Zafar N, Wallace CM, Kieffer P, Schroeder P, Schootman M, Hamvas A. Improving survival of vulnerable infants increases neonatal intensive care unit nosocomial infection rate. Arch Pediatr Adolesc Med 2001;155:1098-1104.

\section{Efficacy of Surveillance for Infection Control in a Surgical Service}

Delgado-Rodriguez and colleagues, from the University of Jaen in Spain, conducted a study to assess the efficacy of surveillance of nosocomial infection (NI) in infection control at a service of general surgery. A surveillance study that included 1,483 patients with a prospective identification of NI was carried out. Its results were discussed with the staff, and a program on NI control was implemented. One year after the pre-intervention study, a similar study that included 1,506 patients was done. The main outcome measure was NI. Incidence rates, incidence rate ratios, crude and multiple-risk factor adjusted for by 